\title{
Discretized disorder in planar semiconductor microcavities: Mosaicity effect on resonant Rayleigh scattering and optical parametric oscillation
}

\author{
Marco Abbarchi, ${ }^{1}$ Carole Diederichs, ${ }^{2}$ Ludovic Largeau, ${ }^{2}$ Vincenzo Ardizzone, ${ }^{2}$ \\ Olivia Mauguin, ${ }^{2}$ Timothee Lecomte, ${ }^{2}$ Aristide Lemaitre, ${ }^{2}$ \\ Jacqueline Bloch, ${ }^{2}$ Philippe Roussignol, ${ }^{2}$ and Jerome Tignon ${ }^{2}$ \\ ${ }^{1}$ Laboratoire Pierre Aigrain, École Normale Supérieure, \\ CNRS (UMR 8551), Université P. et M. Curie, \\ Université D. Diderot, 75231 Paris Cedex 05, France. * \\ ${ }^{2}$ LPN/CNRS, Route de Nozay, F-91460 Marcoussis, France.
}

(Dated: November 19, 2011)

\begin{abstract}
The features of resonant secondary emission by two-dimensional multiple semiconductor microcavities are experimentally investigated. We show that, under normal laser incidence, static disorder determines the final states of the resonant Rayleigh scattering in the high symmetry axes of the GaAs matrix. Scanning transmission electron microscopy reveals a small dislocation density at the layers interfaces and step formation due to strain accumulation and relaxation ruled by the symmetry of the underlying GaAs matrix: this mosaicity effects, a common feature in thick and strained crystals, determines the scattering channels by selecting the crystallographic discretized directions. Moreover, interband optical parametric oscillation of intensity balanced signal and idler beams takes place in the directions selected by the photonic disorder in the distributed Bragg reflector.
\end{abstract}




\section{INTRODUCTION}

Static disorder in semiconductor heterostructures and its features in light-matter interaction ${ }^{1}$ has been the subject of intense research in the past years and it is now well known that the disorder has a strong impact in the physics of multi-layered structures such as quantum wells $(\mathrm{QWs})^{2}$ or two dimensional microcavities (2D-MCs). ${ }^{3}$ The problem of interface roughness received particular attention being a major issue ruling the relaxation of the in-plane translational invariance thus allowing for changes in the wave vector of the exciton/polariton and limiting the coherence. ${ }^{1-4}$

Dielectric fluctuation induced by disorder strongly affect the coherent part of the secondary emission (the resonant Rayleigh scattering, RRS) ${ }^{1,4}$ which, in a first stage, attracted much attention being a very fine probe of potential fluctuation in QWs. ${ }^{1,2,4}$ Later studies put in evidence the features of RRS in QWs coupled to 2D-MCs, highlighting the relevant influence of disorder in the distributed Bragg reflectors. ${ }^{8}$ It is a common opinion that dislocations in the distributed Bragg reflectors dominate the photonic disorder even if direct evidences are lacking. ${ }^{3,5-8}$

In high quality 2D-MCs we can distinguish between two exciton/photon coupling regimes ruling the optical properties of the system: ${ }^{3}$ (i) for small exciton/photon detuning the system may be described in terms of normal modes of coupled oscillators which are called polaritons, half light half matter quasiparticles, and the regime is called strong coupling (SC), (ii) for large exciton/photon detuning, where the SC features are washed out, the emission of the cavity can be described by photonic modes (CMs) and the system is in weak coupling regime (WC). In any case, due to the steep band dispersion of polaritonic or photonic bands, the RRS appears as an annular emission in the far field of the resonant secondary emission. ${ }^{7,8,10}$

In conventional experiments on semiconductor microcavities the excitation of intraband-RRS was obtained only at specific azimuthal angles conditioning the accessible final states and limiting the investigation of disorder related phenomena. ${ }^{8,10}$ In this article we precisely address the origin of photonic disorder in the distributed Bragg reflectors by using a different approach based on multiple 2D-MC (2D-MMC). Thanks to the multiplicity of the CMs, the resonant laser pump can be at normal incidence thus potentially exciting the interband RRS in any accessible in-plane direction. It is the static disorder of the system that chooses a specific elastic scattering channel.

We investigate the far field emission of two 2D-MMCs and its power dependence. At low incident power and large detuning (linear regime) the RRS ring presents strong anisotropies visible as twin speckles in well defined directions, usually parallel to the main crystallographic in-plane 
directions [110] and [1-10]. High-angle annular dark field scanning transmission electron microscopy and X-ray diffraction demonstrate a mosaicity effect ${ }^{9}$ in the structure layers explaining the origin of the very peculiar speckle pattern in the RRS ring. The presence of misfit dislocations is found to play a minor role while the microscopic tilt due to steps formation leading to a broadening of the X-Ray diffraction pattern is the main source of disorder.

The study of the secondary emission in the high excitation regime and small detuning (nonlinear regime) shows the onset of optical parametric oscillation seeded by the RRS in the same directions imposed by underlying lattice geometry. Nevertheless, other directions for the OPO are possible when different kind of defects are present.

The paper is organized as flows: in Section II we give a short description of the samples in analysis and of the experimental arrangement; in Section II we show the experimental results of spectroscopic and structural investigation by RRS, STEM and X-Ray diffraction imaging; in Section IV we draw the conclusions.

\section{SAMPLE GROWTH AND EXPERIMENTAL METHODS}

Two 2D-MMCs samples, labeled as A and B, are grown in a molecular beam epitaxy reactor. A cross section of sample A obtained by high-angle annular dark field scanning transmission electron microscopy (HAADF-STEM images are registered with a IEOF 220FS microscope) is shown in Fig.1 (a) while a magnified view of the central cavity is shown Fig.1 (b). ${ }^{11}$ Three GaAs $\lambda$-cavities (MC1, MC2 and MC3 from top to bottom) are embedded between GaAs/AlAs distributed Bragg reflectors and are separated by equal intermediate distributed Bragg reflectors. The number of GaAs/AlAs layers in the distributed Bragg reflectors from bottom to top is 15/11.5/11.5/18.5 for sample A and 13/13.5/13.5/13 for sample B bringing to different Q factor (1350 for sample A and 740 for sample B). In sample A (sample B) nine (one) $\mathrm{In}_{0.07} \mathrm{Ga}_{0.93} \mathrm{As}$ QWs [width $8.5 \mathrm{~nm} \pm 0.3$ $\mathrm{nm}$ ] are placed at the antinodes of the electromagnetic field determining the excitonic transition at $\sim 1.468 \mathrm{eV}(\sim 1.457 \mathrm{eV})$ at $6 \mathrm{~K}$. A scheme of the sample is shown in Fig.1 (c) together with the experimental implementation of spectroscopic characterization. A wedge in MC1 and MC3 thickness (the angles $\alpha$ and $\beta$ in Fig.1 (a)) is introduced in order to control the relative energies of the individual CMs and of the detuning $\delta$ with respect to the excitonic transition (here we define the detuning $\delta$ as the energy difference between the excitonic transition and the energy minimum of the lowest cavity mode: $\left.\delta=\mathrm{E}_{X}-\mathrm{E}_{1}(k=0)\right)$. For relatively small $\delta$, in SC regime, 
the measured vacuum Rabi splitting is $\sim 10.4 \mathrm{meV}$ for sample A and $\sim 3.6 \mathrm{meV}$ for sample B. The dispersion in energy-momentum space of coupled photonic/polaritonic bands is represented in Fig.1 (d). The characterization of bands dispersion in energy momentum space of the 2D-MMC under non-resonant excitation (not shown) ${ }^{11}$ demonstrates the presence of three photonic CMs (labeled as 1, 2 and 3 in Fig. 1 (c)).

The CW excitation is provided by a tunable Ti:Sapphire laser focused normal to the sample surface (see Fig.1 (c)) on a spot of $\sim 40 \mu \mathrm{m}$ (full width at half maximum, FWHM). The laser polarization is set linear. The sample is cooled down to $6 \mathrm{~K}$ in a cold-finger, liquid-Helium cryostat. The secondary emission collected in the far field is discriminated in polarization before detection.

By exciting interband energy degenerate scattering processes $2(0,0) \rightarrow 1(+k,-k)$ from the bottom of band 2 at $k_{\|}=0$ we probe all the possible in-plane final states belonging to band 1 (see Fig.1 (c) and (d) where the possible final states for the RRS are represented by a isoenergetic ring, while specific scattering channels are represented by dots labeled as $\mathrm{R}$ on the ring). Note that in the following we will deal only with MC1 and MC2 and we will neglect MC3 which does not play any role in this experiment.

Differently from state-of-the-art RRS experiments in 2D-MCs the present system offers the big advantage of normal incidence excitation. Besides a simpler implementation of the experimental setup, this distinctive property of 2D-MMCs is essential for the study of RRS since it allows exciting along a high symmetry axis of the system (parallel to the crystallographic growth direction [001] as shown in Fig.1 (c)). Most importantly, this kind of experimental configuration represents an isotropic probe producing interband RRS and sampling the full extension of the elastic ring (see Fig.1 (c), and (d)). Fig.1 (c) describes the experimental arrangement: the collection of transmitted secondary emission is made in the far field by imaging the RRS ring on a silicon based CCD camera. Moreover, by selecting a single speckle (labeled as R in Fig.1 (c), and (d)) in the far field with an iris, we can measure the transmitted RRS intensity with a silicon based photodiode for a quantitative analysis. We choose a reference frame where the crystallographic directions [1-10] and [110] corresponds to $\phi=0$ and $\phi=90$ degrees, respectively (see Fig. 1 (c) and (d). 


\section{EXPERIMENTAL RESULTS AND DISCUSSION}

\section{A. RESONANT RAYLEIGH SCATTERING}

Fig.2 (a),(b) and (c) show the 2D-MMC far field emission pattern for different detunings $\delta$ under resonant excitation of mode 2 at $k_{\|}=0$ in the linear regime (excitation power $\sim \mathrm{KW} / \mathrm{cm}^{2}$ ). The strong emission at $\theta=0$ degrees is laser light transmitted through the sample. Fig.2 (c) show a typical power dependence of a single speckle (that one at $\phi=90$ degrees in Fig.2 (c)).

Distinctive features of the detected secondary emission in sample A and B are summarized as follows: (i) the secondary emission shows a polarization parallel to that of the incident laser, (ii) in all the investigated conditions the ring-like emission (the aperture is generally between $\theta \simeq 12$ and $\theta \simeq 15$ degrees in the investigated conditions) is anisotropic and structured in a speckle pattern, (iii) the intensity of a single speckle increases linearly with respect to the incident laser power (see the linear fit to data in Fig.2 (d)). Based on these evidences we interpret the resonant secondary emission as RRS induced by static disorder in the 2D-MMC. These results, which are obtained for large negative detuning $(\delta \ll 0)$ where the modes are prevalently photonics, account for a relevant contribution to the disorder in the distributed Bragg reflectors.

The speckles pattern is centrally symmetric: for each speckle at a definite angle $\phi$ a twin speckle is present at $\phi+180$ degrees (as highlighted by circles of same color around two twin speckles in Fig.2 (a), (b) and (c)). Most important, the directions identified by two twin speckles which recurrently appear in several measurements and in different experimental conditions, are not randomly distributed all along the RRS ring but tend to coalesce in specific in-plane directions: the brightest speckles appear in correspondence of the directions of the high symmetry axes of the GaAs matrix (i.e. [110] and [1-10]). At margin we note that different directions of the RRS are possible as shown in Fig. 2 (b), where the speckles with lower intensities at $\phi=45$ and 225 degrees correspond to the crystallographic direction [100]. We conclude that disorder in the Bragg mirrors is discretized and is influenced by the same symmetry of the underlying GaAs matrix.

\section{B. STRUCTURAL CHARACTERIZATION}

In order to precisely address and clarify the origin of the disorder responsible for the RRS we performed TEM and STEM at the layers interface and high resolution X-ray diffraction measurements with a triple axis PANalytical XPert Pro diffractometer. Fig. 3 (a) is a 220 dark field TEM 
image where a dislocation is visible at the first interface substrate/DBR while Fig. 3 (b) is a high resolution view of the defect. This is the result of plastic relaxation inducing a misfit dislocation which in turn results in a threading dislocation (not visible in the plane of view) propagating across the microcavity. The density of this dislocation ( $\sim 1$ dislocation every $3 \mu \mathrm{m})$ is much smaller than that expected for a completely relaxed structure $(\sim 1$ dislocation every $250 \mathrm{~nm})$ suggesting that the main mechanism of strain relaxation is not due to nucleation of dislocations.

Fig. 3 (c), (d) and (e) show HAADF-STEM images of the substrate/DBR interfaces (vertical and horizontal axes in Fig. 3 (d) and (e) are intentionally rescaled in order to highlight the sharp features). The periodicity of the ondulation is hundreds of nanometers and, as we show below, this periodic mosaicity is also observed in X-ray diffraction measurements. This effect is the result step formation and bunching due to elastic accommodation of the strain.

Typical diffraction reciprocal space mapping (RSM) around the 004 reflection obtained by high resolution X-ray diffraction measurements is shown in 3 (d). The RSM is shown in the reciprocal lattice units $\left(\AA^{-1}\right)$. The horizontal $\mathrm{Q}_{x}$ axis is parallel to the [001] growth direction and the vertical $\mathrm{Q}_{y}$ axis is parallel to the [110] in-plane direction. The periodic peaks correspond to diffraction of the distributed Bragg reflectors periodic superlattice epitaxial layers, while the nonperiodic peak correspond to the 004 diffraction of the substrate. The peaks broadening, due to slight deviations of the 004 reciprocal space vector is associated to a tilt of the crystallographic planes forming misoriented domains ${ }^{9}$. The formation of steps at the interfaces induces the tilt of the crystallographic planes resulting in misoriented blocks, domains comparable to a mosaic. From the peaks full width at half maximum measured on four RSMs, we estimate a coherence length of hundreds of nanometers. The coherence length as deduced by the RSM packs broadening is $\sim 500$ $\mathrm{nm}$ and $\sim 800 \mathrm{~nm}$ along the preferential [110] and [1-10] in-plane directions for sample A, while it is $\sim 400 \mathrm{~nm}$ for both directions in sample B.

This structural long-range disorder is typical of thick and strained crystals such as 2D-MCs. From the comparison with HAADF-STEM analysis we conclude that the main scattering mechanism is associated to microscopic tilts, in fact the distances deduced by the RSM are consistent with the ondulations shown in 3 (c), much smaller than the distance between misfit dislocations. We conclude that the plastic relaxation is the main source of disorder in the distributed Bragg reflectors and justify the peculiar far field pattern observed in the RRS ring. 


\section{OPTICAL PARAMETRIC OSCILLATION}

Besides disorder related phenomena, a major subject of investigation in 2D-MCs is the generation of optical parametric oscillation. ${ }^{11-16}$ It is now well established that strong $\chi^{3}$-polaritonic/excitonic non-linearity can give rise to parametric phenomena ${ }^{11-16}$ and that intensity balanced signal and idler ( $\mathrm{S}$ and I) beams can be produced in energy degenerate interband processes under normal incidence excitation. ${ }^{18}$ For this reason we performed measurements of resonant secondary emission at small detuning under high incident power (nonlinear regime).

In the same experimental conditions previously described, we investigate the far field of twin speckles for $\delta \simeq 0 \mathrm{meV}$ in sample A. As previously shown for the linear excitation regime, the far field pattern shows strong anisotropies (see Fig. 4 (a)) being characterized by twin speckles in the directions [110] and [1-10]. The power dependence shows a threshold like trend: below a threshold power $\left(\mathrm{P}_{T h}=2 \mathrm{KW} / \mathrm{cm}^{2}\right)$ the emission is dominated by incoherent secondary emission and RRS while after the threshold the emission intensity abruptly changes its slope with a nonlinear trend. This is the signature of the onset of interband optical parametric oscillation as already reported in previous works. ${ }^{11,18}$ Here, the resonant excitation of interband parametric scattering of two incident photons at $k_{\|}=0$ is ruled by the phase matching conditions: $\mathrm{S}$ and $\mathrm{I}$ are degenerate in energy with respect to the pump which is resonant with the bottom band 2(0,0) and have opposite in-plane momentum, coincident with the crystallographic directions. ${ }^{18}$

On the base of these evidences we conclude that the same disorder in the distributed Bragg reflectors responsible for the RRS determines the possible final states of parametric scattering and optical parametric oscillation. This implies that a specific mode of the coherent oscillation of $\mathrm{S}$ and I is seeded by the RRS scattering and stabilized in a well defined mode, avoiding undesired mode hopping and reducing the oscillation threshold to very small values differently from a perfectly isotropic system.

We stress that all these features can be easily evidenced thanks to the symmetric configuration of excitation which does not condition the final states of the RRS or parametric scattering. We mention that the influence of directional static disorder in intraband optical parametric oscillation has already been reported in real-space imaging experiments, suggesting a strain relaxation due to the lattice mismatch of the AlAs layers in the distributed Bragg reflectors ${ }^{16}$ in analogy with similar evidences in RRS experiments. ${ }^{8,17}$ The dominant source of disorder was believed to be misfit dislocations in the thick and strained distributed Bragg reflectors. 
For the sake of thoroughness we report a last case of directional disorder influencing the optical parametric oscillation eventually found when addressing specific points on sample B.

Despite the high optical quality of the investigated samples, real space imaging of resonant transmission (see the inset of Fig. 5 (a)) occasionally reveals the presence of long line-shaped defects. When focusing the excitation on this kind of defects the corresponding far field emission shows, besides the usual [110] and [1-10] directions determined by the mosaicity, much stronger emission in directions different from the crystallographic axes (see a representative example in Fig. 5 (a) where S and I at $\sim 127-307$ degrees are shown). Power dependence measurements on $\mathrm{S}$ and I (see Fig. 5 (b)) show the onset of optical parametric oscillation suggesting that sources of disorder other than mosaicity dominate determining the final states of the scattering channels. Tentatively, we attribute this kind of disorder to surface defects or threading dislocations.

We note that the parametric nature of the two-photon process agrees well with theoretical predictions and experimental evidences: ${ }^{12,13}$ at low excitation power the twin beam intensity increases quadratically with the incident power and at a threshold value abruptly changes its slope, following a square root dependence (see the quadratic and square-root fit to data in the inset of Fig. 5 (b)). Note that threshold power $\mathrm{P}_{T h}$ for OPO increases when going to negative detuning. ${ }^{19}$ This is why we only observe RRS in Fig.2 (at very large negative detuning, $\delta \ll 0$ ) and for similar excitation powers OPO in Fig.4 and 5 (at zero detuning, $\delta=0$ ). ${ }^{12}$ Most important, $\mathrm{S}$ and I beams are intrinsically balanced in intensity, a very attractive feature for quantum optics applications of parametric scattering and optical parametric oscillation in semiconductor nanostructures. ${ }^{18,20}$.

\section{CONCLUSION}

In this paper we have shown that a mosaicity effect in the distributed Bragg reflectors rules the disorder in 2D-MCs and strongly affects the far field features of the secondary emission, the main source of disorder being a plastic accommodation of the strain. The accumulation and relaxation of strain along the high symmetry axes of the crystal determines the RRS along the same directions in different conditions of exciton-photon detuning and/or coupling. In the nonlinear excitation regime intensity balanced optical parametric oscillation of $\mathrm{S}$ and I beams takes place along the same crystallographic directions being seeded in specific modes by the directional RRS. Nevertheless, other directions are accessible to the OPO when the defectivity determines a strong anisotropy in a defined scattering channel. 
In conclusion we remark that the recent quality improvement in the fabrication techniques of epitaxial materials enabled the demonstration of surprising quantum effects in polariton physics. Striking phenomena such as polariton condensation ${ }^{21}$ and superfluidity ${ }^{7}$, quantized vortices ${ }^{22}$ and solitons formation, ${ }^{23}$ Josephson oscillations ${ }^{24}$ as well as parametric scattering phenomena ${ }^{11-16,18}$ have been recently shown. If by one side the high optical quality of the microcavities relies in the reduced defectivity detrimental for the radiative emission properties, on the other side the presence of residual disorder is at the base of the observation of the mentioned effects. In this sense the study of the ultimate sources of disorder in microcavities can help the understanding and the exploitation of these peculiar phenomena.

\section{ACKNOWLEDGMENTS}

We thank Alberto Amo for fruitful discussions. M. A. and V. A. thank the European project EU Network ITN Clermont-4.

1 J. Hegarty, M. D. Sturge, C. Weisbuch, A. C. Gossard, and W. Wiegmann, Phys. Rev. Lett. 49, 930 (1982). M. Gurioli, F. Bogani, S. Ceccherini, and M. Colocci, ibid. 78, 3205 (1997); X. Marie, P. Le Jeune, T. Amand, M. Brousseau, J. Barrau, and M. Paillard ibid. 79, 3222 (1997); S. Haacke, R. A. Taylor, R. Zimmermann, I. Bar-Joseph, and B. Deveaud, ibid. 78, 2228 (1997); M. Woerner J. Shah, ibid. 81, 4208 (1998). D. Birkedal and J. Shah, ibid. 81, 2372 (1998).

2 Solid State Physics, Excitons in Semiconductors Nanostructures Vol. 57 E. Runge (Academic, New York, 2002); Semiconductor optics C. Klingshirn (2nd ed., Springer-Verlag, 2005).

3 J. Baumberg and L. Vina, Semicond. Sci. Technol. 18, S279 (2003); A. V. Kavokin, J. J. Baumberg, G. Malpuech, and F. P. Laussy, Microcavities (Oxford University Press, New York, 2007).

4 W. Langbein, J. M. Hvam, and R. Zimmermann, Phys. Rev. Lett. 3205 82, 1040 (1999); P. Bozsoki, P. Thomas, M. Kira, W. Hoyer, T. Meier, S.W. Koch, K. Maschke, I. Varga, and H. Stolz, ibid 97, 227402 (2006).

5 A. V. Shchegrov, D. Birkedal, and J. Shah, Phys. Rev. Lett 84, 3478 (2000); F. M. Marchetti, J. Keeling, M. H. Szymanska, and P. B. Littlewood, ibid. 96, 066405 (2006). 
6 M. M. Glazov and L. E. Golub, Phys. Rev. B 77, 165341 (2008); T. C. H. Liew, C. Leyder, A. V. Kavokin, A. Amo, J. Lefrere, E. Giacobino, and A. Bramati ibid. 79, 125314 (2009); M. M. Glazov and L. E. Golub, ibid. 82, 085315 (2010).

7 I. Carusotto and Cristiano Ciuti, Phys. Rev. Lett. 93, 166401 (2004); A. Amo, D. Sanvitto, F. P. Laussy, D. Ballarini, E. del Valle, M. D. Martin, A. Lematre, J. Bloch, D. N. Krizhanovskii, M. S. Skolnick, C. Tejedor, and L. Vina,, Nat. 457, 291 (2009).

8 M. Gurioli F. Bogani, D. S. Wiersma, Ph. Roussignol and G. Cassabois, G. Khitrova and H. Gibbs, Phys. Rev. B 64, 165309 (2001); W. Langbein, and Jorn M. Hvam, Phys. Rev. Lett. 88, 047401 (2002); W. Langbein, J. Phys. Condens. Matter 16 S3645 (2004); M. Gurioli, F. Bogani, L. Cavigli, H. Gibbs, G. Khitrova, and D. S. Wiersma, Phys. Rev. Lett. 94, 183901 (2005); W. Langbein, Rivista del Nuovo Cimento 33, 255 (2010).

9 V. Holy, J. Kubena, E. Abramof, K. Lischka, A. Pesek, and E. Koppensteiner, J. Appl. Phys. 741736 (1993).

10 T. Freixanet B. Sermage J. Bloch, J. Y. Marzin, and R. Planel, Phys. Rev. B 60, R8509 (1999); D. M. Whittaker, ibid. 61, R2433 (2000); P. G. Savvidis, J. J. Baumberg, D. Porras, D. M. Whittaker, M. S. Skolnick, and J. S. Roberts, ibid. 65, 073309 (2002) M. Richard, J. Kasprzak, R. Romestain, R. Andre, and Le Si Dang, Phys. Rev. Lett. 94, 187401 (2005).

11 C. Diederichs and J. Tignon, Appl. Phys. Lett. 87, 251107 (2005) C. Diederichs, J. Tignon, G. Dasbach, C. Ciuti, A. Lematre, J. Bloch, Ph. Roussignol, and C. Delalande, Nat. 440, 904 (2006)

12 C. Ciuti, P. Schwendimann, B. Deveaud, and A. Quattropani, Phys. Rev. B 62, R4825 (2000); C. Ciuti, ibid. 63, 041303(R) (2001); D. M. Whittaker, ibid. 63, 193305 (2001); C. Ciuti, P. Schwendimann, and A. Quattropani, Semicon. Sci. Tech. 18, S279 (2003); J. Ph. Karr, A. Baas, and E. Giacobino, Phys. Rev. A 69, 063807 (2004); A. Baas et al., Phys. Rev. A 69, 023809 (2004); D. Taj T. Lecomte, C. Diederichs, Ph. Roussignol, C. Delalande, and J. Tignon, Phys. Rev. B 80, 081308 (2009).

13 R. Butte, M. S. Skolnick, D. M. Whittaker, D. Bajoni, and J. S. Roberts, Phys. Rev. B 68, 115325 (2003)

14 Y. R. Shen, The Principles of Nonlinear Optics Wiley Inter- Science, Hoboken, (2003)

15 J. J. Baumberg, P. G. Savvidis, R. M. Stevenson, A. I. Tartakovskii, M. S. Skolnick, D. M. Whittaker, and J. S. Roberts, Phys. Rev. B 62, R16247 (2000); R. Houdre, C. Weisbuch, R. P. Stanley, U. Oesterle, and M. Ilegems, Phys. Rev. Lett. 85, 27932796 (2000); M. Saba, C. Ciuti, J. Bloch, V. Thierry-Mieg, R. Andrea, Le Si Dang, S. Kundermann, A. Mura, G. Bongiovanni, J. L. Staehli, B. Deveaud, Nat. 414, 731 (2003); W. Langbein, Phys. Rev B 70, 205301 (2004); D. N. Krizhanovskii, D. Sanvitto, A. P. D. Love, 
M. S. Skolnick, D. M. Whittaker, and J. S. Roberts , Phys. Rev. Lett. 97, 097402 (2006); M. Romanelli, C. Leyder, J. Ph. Karr, E. Giacobino, and A. Bramati, Phys. Rev. Lett. 98, 106401 (2007).

16 D. Sanvitto, D. N. Krizhanovskii, D. M. Whittaker, S. Ceccarelli, M. S. Skolnick, J. S. Roberts, Phys. Rev. B 73, 241308(R) (2006).

17 M. Gurioli, F. Bogani, D. S. Wiersma, Ph. Roussignol, G. Cassabois, G. Khitrova, and H. Gibbs,, phys. stat. sol. (a) 190, 363 (2002)

18 M. Abbarchi, V. Ardizzone, T. Lecomte, A. Lematre, I. Sagnes, P. Senellart, J. Bloch, Ph. Roussignol, and J. Tignon, Phys. Rev. B 83, 201310(R) (2011)

19 A detailed discussion of this topic is beyond the aim on the present investigation. A paper on this subject is in preparation.

20 K. Edamatsu, G. Oohata, R. Shimizu, and T. Itoh, (London) 431, 167 (2004); A. Baas, J.-Ph. Karr, M. Romanelli, A. Bramati, and E. Giacobino, Phys. Rev. Lett. 96, 176401 (2006); S. Portolan, O. Di Stefano, S. Savasta, F. Rossi,3 and R. Girlanda, Phys. Rev. B 77, 035433 (2008); S. Portolan, O. Di Stefano, S. Savasta, F. Rossi,3 and R. Girlanda, ibid. 77, 195305 (2008); J. U. Fürst, D.V. Strekalov, D. Elser, A. Aiello, U. L. Andersen, Ch. Marquardt, and G. Leuchs, Phys. Rev. Lett. 106, 113901 (2011).

21 J. Kasprzak, M. Richard, S. Kundermann, A. Baas, P. Jeambrun, J. M. J. Keeling, F. M. Marchetti, M. H. Szymanska, R. Andre, J. L. Staehli, V. Savona, P. B. Littlewood, B. Deveaud, and Le Si Dang, Nat. 443, 409 (2006); D. Sanvitto, A. Amo, L. Via, R. Andr, D. Solnyshkov, and G. Malpuech Phys. Rev. B 80, 045301 (2009)

22 K. G. Lagoudakis, M. Wouters, M. Richard, A. Baas, I. Carusotto, R. Andre, D. Le Si Dang, and B. Deveaud-Peledran Nat. Phys. 4, 706710 (2008); K. G. Lagoudakis, T. Ostatnicky, A. V. Kavokin, Y. G. Rubo, R. Andre, and B. Deveaud-Pledran, Science 326, 974 (2009)

23 A. Amo, S. Pigeon, D. Sanvitto, V. G. Sala, R. Hivet, I. Carusotto, F. Pisanello, G. Lemenager, R. Houdre, E Giacobino, C. Ciuti, and A. Bramati, Science 332, 1167 (2011); H. Flayac, D. D. Solnyshkov, and G. Malpuech, Phys. Rev. B 83, 193305 (2011)

24 K. G. Lagoudakis, B. Pietka, M. Wouters, R. Andre, and B. Deveaud-Pledran, Phys. Rev. Lett. 105, 120403 (2010)

\section{FIGURES}




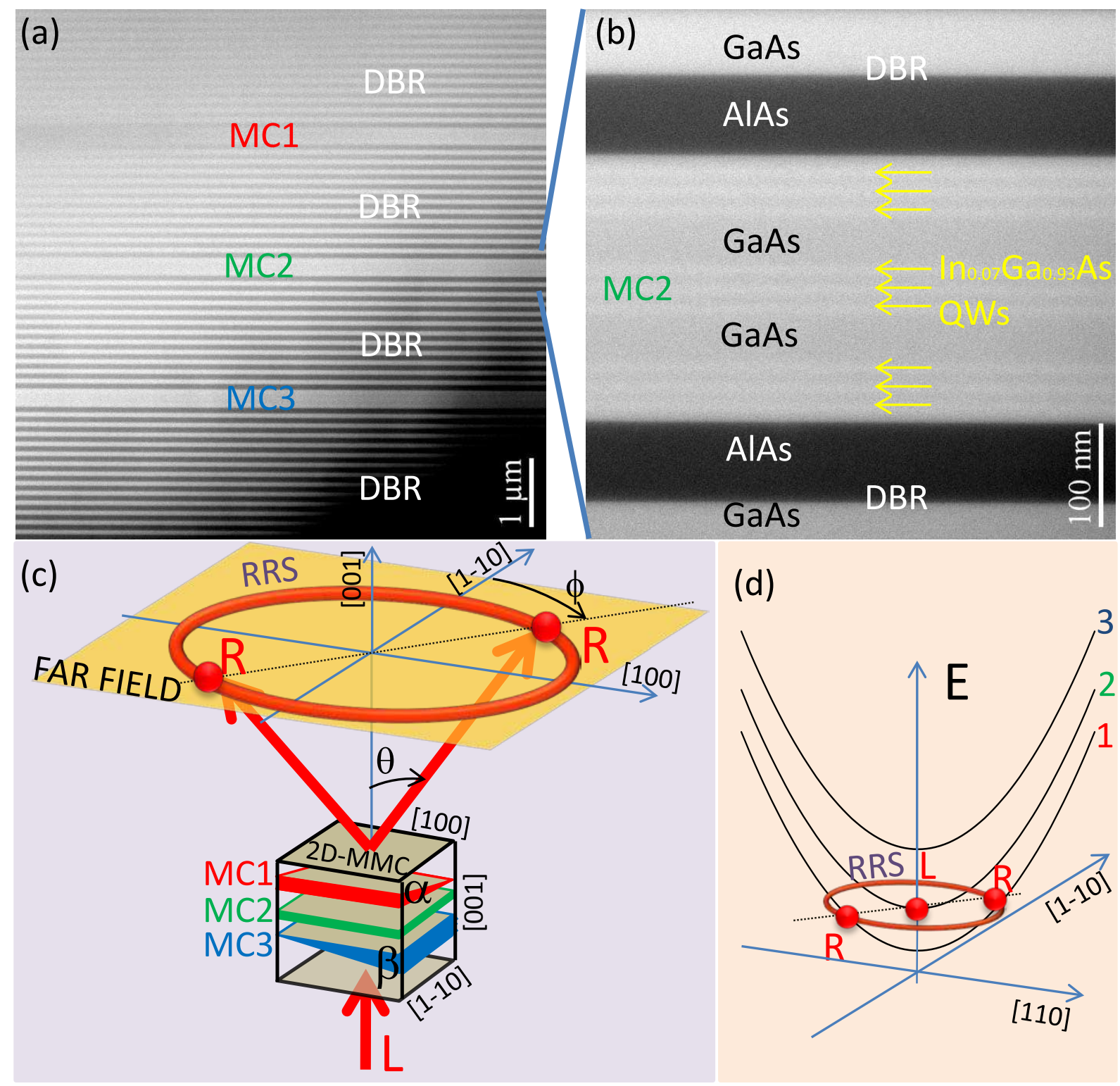

FIG. 1. (Color online) (a) HAADF-STEM image of sample A. (b) Magnified view of MC1 of sample A. The different layers are labeled, the yellow arrows indicate the $\operatorname{In}_{0.07} \mathrm{Ga}_{0.93}$ As QWs. (c) Scheme of 2D-MMC (the distributed Bragg reflectors are not represented) and of the experimental configuration of excitation/detection of the far field. The angles $\alpha$ and $\beta$ represent the cavity wedges. (d) Cavity modes/lower polariton states dispersions in energy/momentum space. The circle represents the possible final states of the RRS while dots and arrows specify a particular scattering processes $2(0,0) \rightarrow 1(+k,-k)$ labeled as R. 

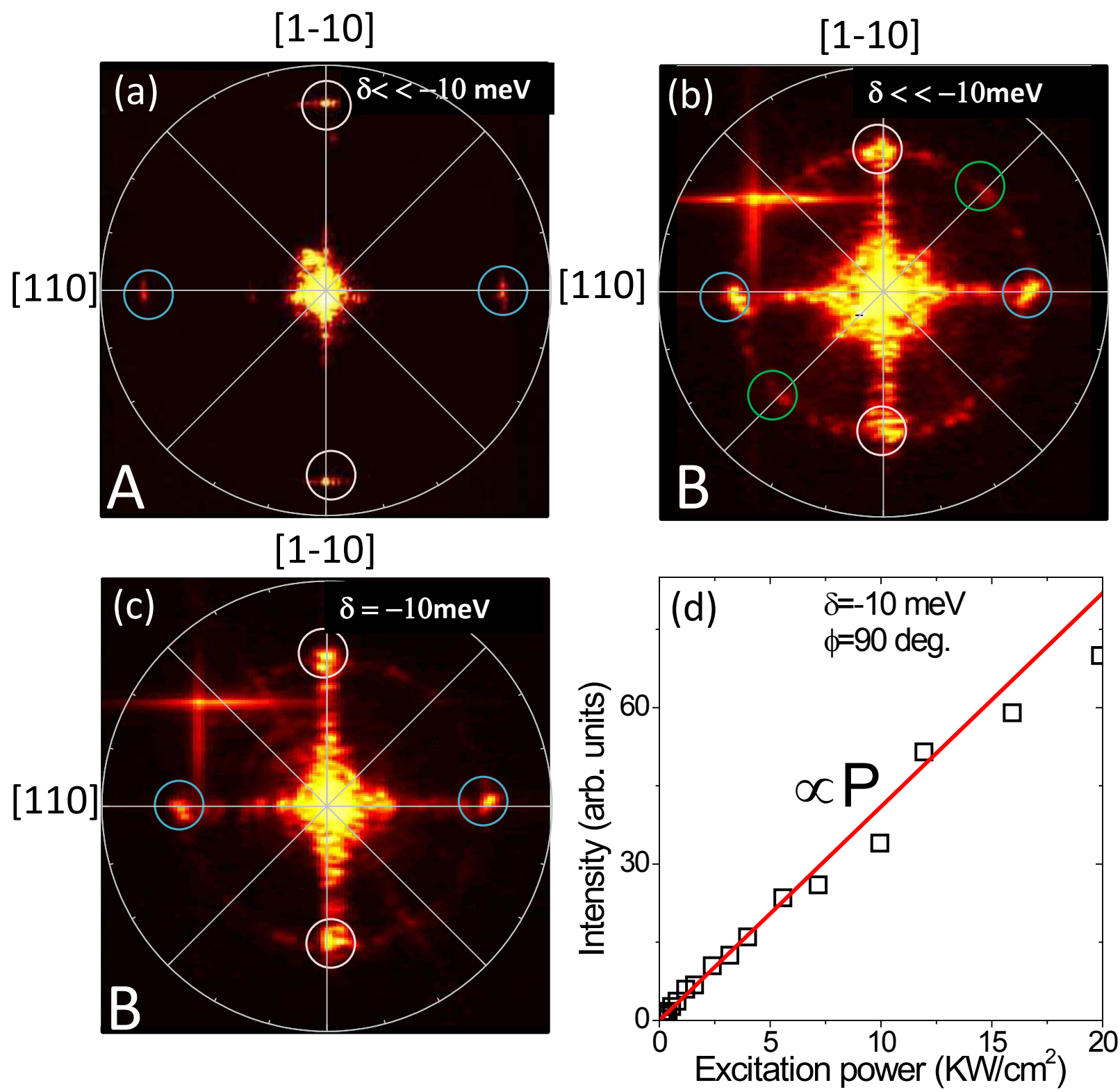

FIG. 2. (Color online) RRS ring from sample A (a), and sample B (b) and (c) as measured in the process $2(0,0) \rightarrow 1(+k,-k)$ for different $\delta$ and low incident power. The cross-shaped emission at $\phi \simeq 315$ degrees in graphs (b) and (c) is stray light entering in the system. (d) Symbols: intensity of the speckle at $\phi=90$ degrees of graph (c) as a function of the incident power. The line is a linear fit to data. 

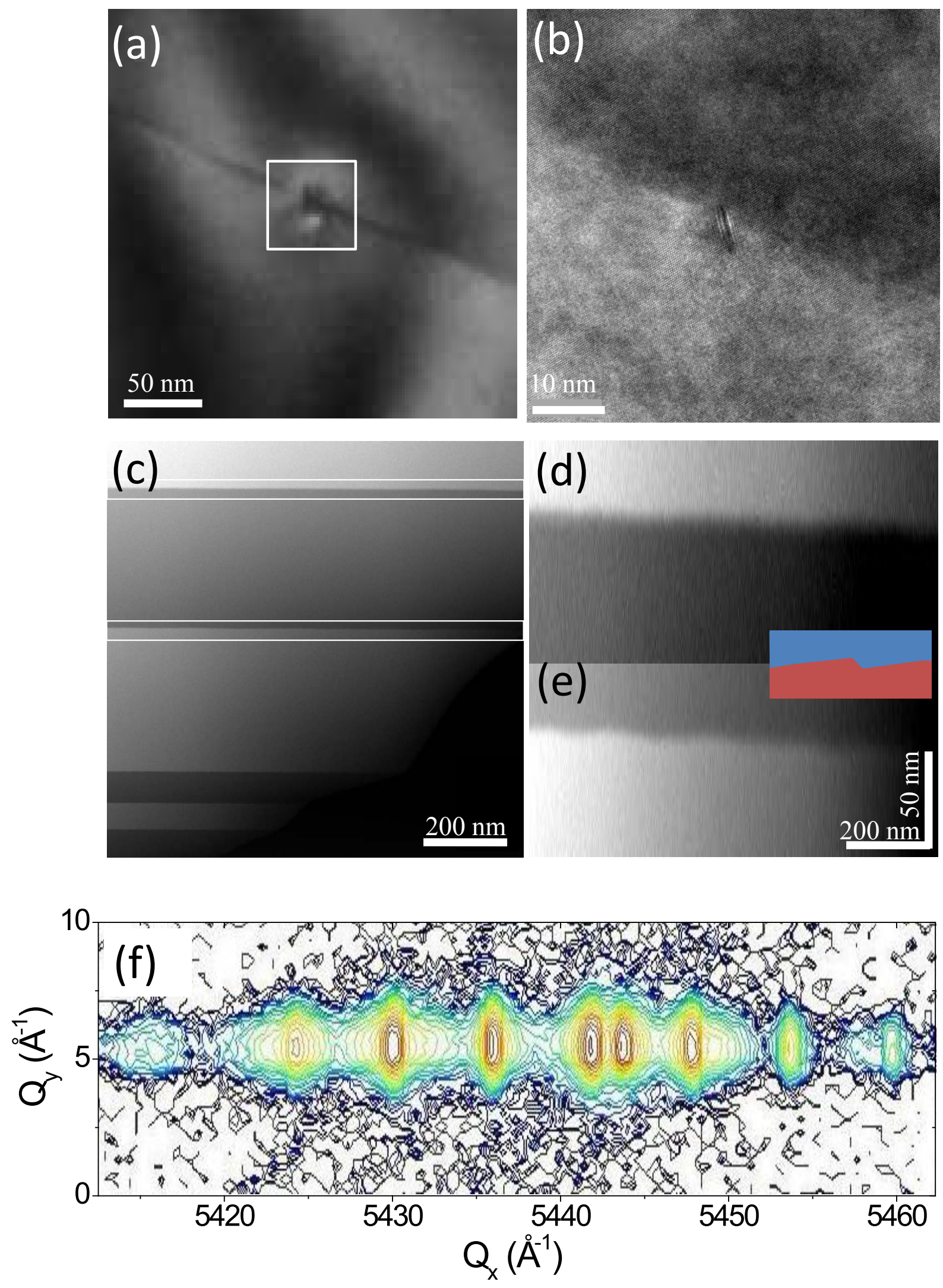
FIG. 3. (Colour online) (a) 220 Dark Field TEM image of sample A at the first interface substrate/DBR. The white square indicate the area shown in graph (b). (b) High resolution TEM image of the misfit dislocation shown in (a). (c) HAADF-STEM image of cavity A at the first interface substrate/DBR. The horizontal white lines indicate the areas shown in graph (d) and (e). (d) and (e) Expanded view of the areas selected in graph (c). Vertical and horizontal axes have different scales in order to evidence the layer tilts. The inset shows a scheme of the layers arrangement. (f) High resolution X-Ray reciprocal space mapping of sample $\mathrm{B}$ around the 004 reflection.
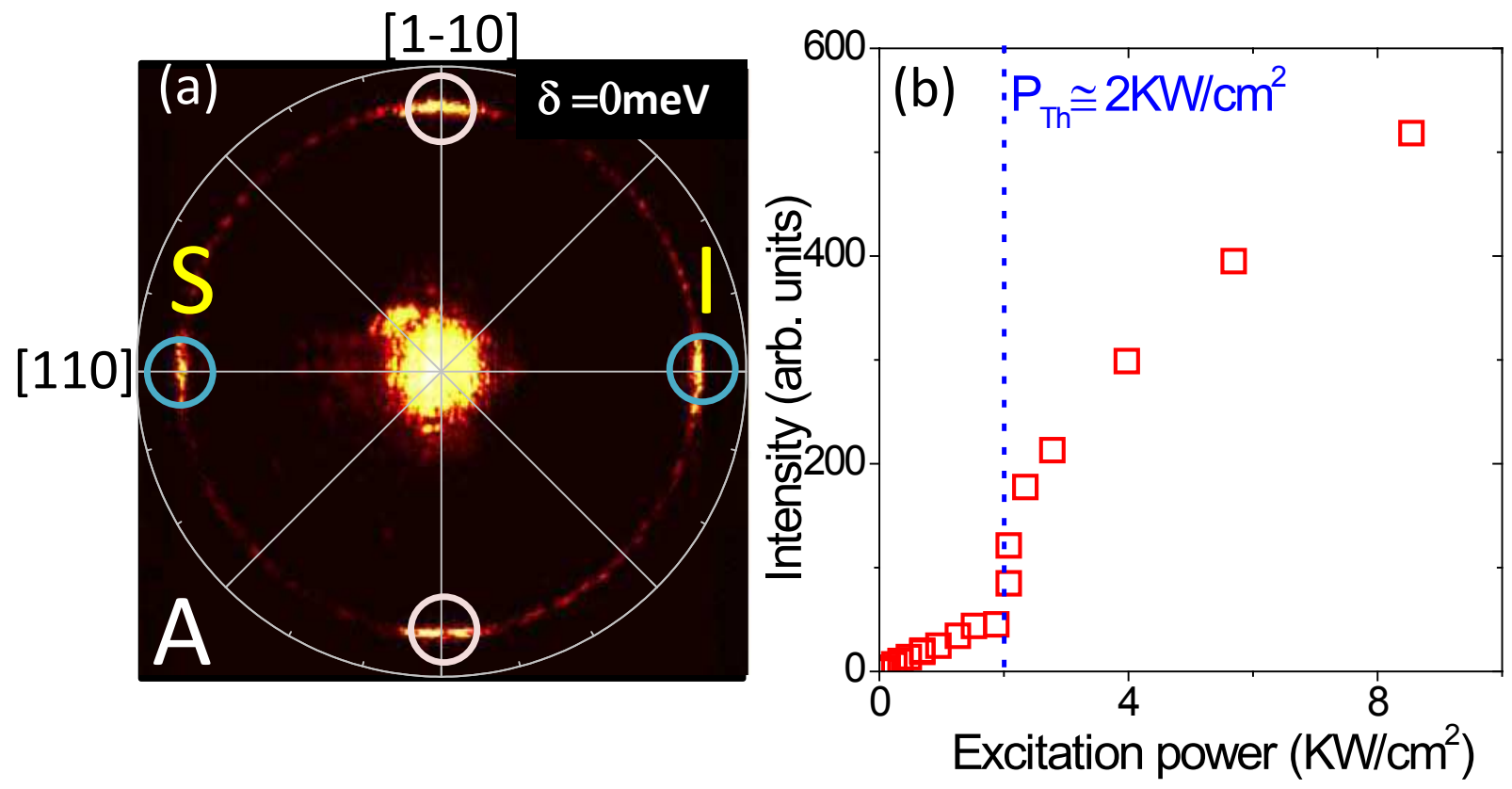

FIG. 4. (Colour online) (a) far field pattern for the interband scattering $2(0,0) \rightarrow 1(+k,-k)$ under high excitation power for sample A. (b) Symbols: $\mathrm{S}$ intensity as a function of the incident power. The vertical dashed line labels the threshold power $\mathrm{P}_{T h}$. 


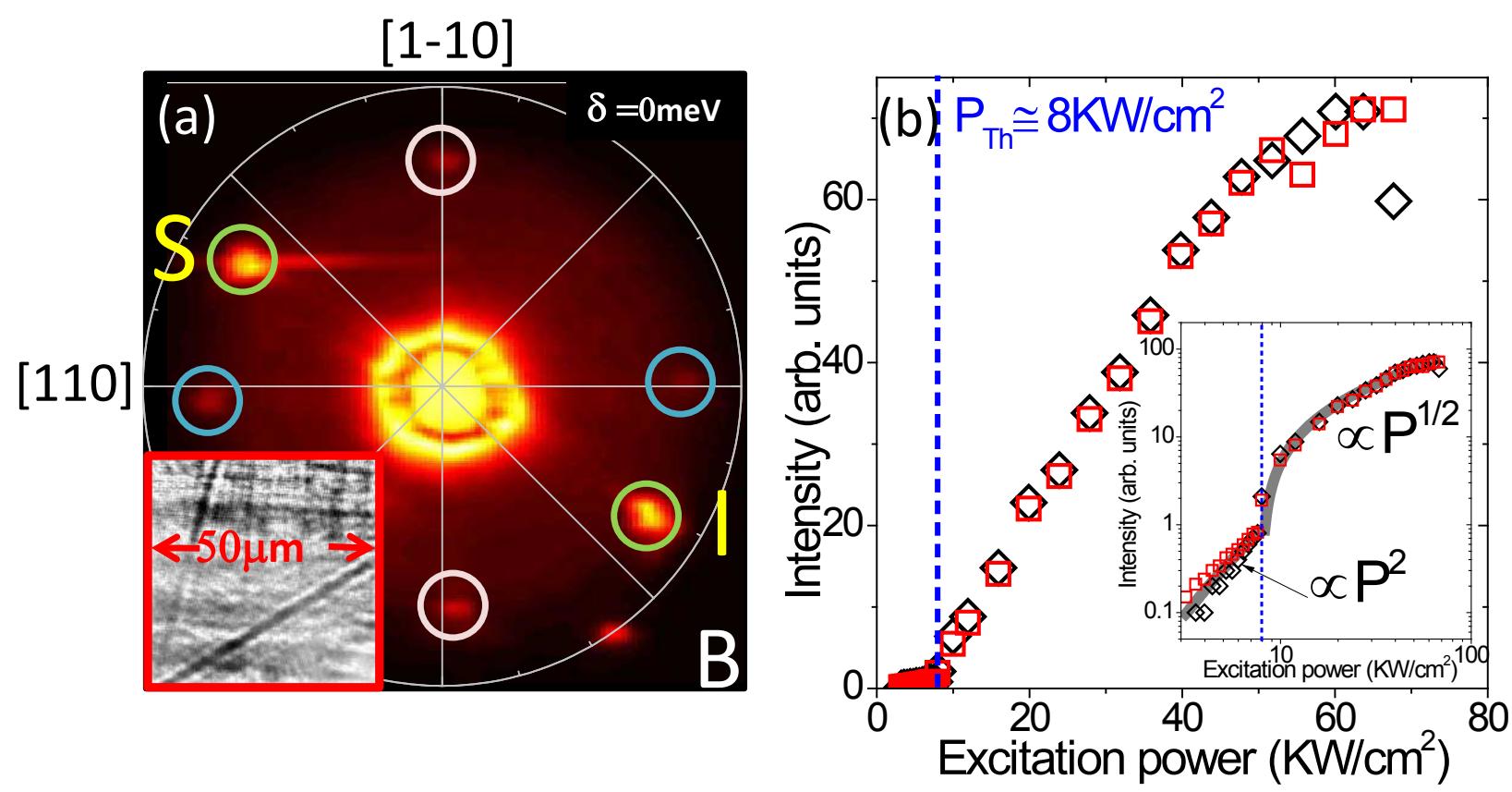

FIG. 5. (Color online) (a): far field pattern for the interband scattering $2(0,0) \rightarrow 1(+k,-k)$ under high excitation power for sample B. Inset: $50 \mu \mathrm{m} \times 50 \mu \mathrm{m}$ real-space image of resonant transmission. (b) Symbols: S and I intensity as a function of the incident power. The vertical dashed line labels the threshold power $\mathrm{P}_{T h}$. Inset: same as (b) in logarithmic scales. Lines are quadratic (below $\mathrm{P}_{T h}$ ) and square-root (over $\left.\mathrm{P}_{T h}\right)$ fit to data. 